\section{Depression and Culture in the TOP DD Network Study: Assessing Depressive Symptoms, Self- harm, and Hospitalization among Dissociative Patients across Four Cultures}

\section{Abstract}

Depressive symptoms, Non-suicidal Self-injury (NSSI), and suicide attempts are common and often require intensive interventions among individuals with complex Dissociative Disorders (DDs). Most of the treatment research on DDs has been conducted in the United States. The present study examined depressive symptoms and disorders, NSSI, suicide attempts, and psychiatric hospitalizations among DD patients participating in outpatient treatment from the United States, Canada, Australia, and Norway. Analyses showed that patients from the United States and Canada had fewer lifetime and recent hospitalizations compared to Norwegian and Australian DD patients. There were no cross-cultural differences in suicide attempts, NSSI, depressive symptoms, or types of depressive disorders. Our findings regarding rates of hospitalization are likely due to cross-cultural differences in health care utilization and systems, rather than to phenomenology related to depression. The relative uniformity of these symptoms and depressive disorders across cultures may indicate that dissociation, and possibly trauma, has a consistent link with depressive symptomatology regardless of one's culture. We discuss the implications for training clinicians and future research.

Keywords: Depression; Psychiatric; Dissociative Disorders (DDs); Posttraumatic stress disorder

Received: June 13, 2017; Accepted: June 16, 2017; Published: June 30, 2017

\section{Aliya R Webermann ${ }^{1}$, Bethany L Brand ${ }^{2 *}$, Hugo J Schielke ${ }^{3}$, Shaina A Kumar² and Amie C Myrick ${ }^{4}$}

1 University of Maryland, Baltimore County, Baltimore, MD, USA

2 Psychology Department, Towson University, Towson, MD, USA

3 California Department of State Hospitals, Napa, CA, USA

4 Family and Children's Services, Bel Air, MD, USA

*Corresponding author: Bethany L Brand

” bbrand@towson.edu

Professor, Psychology Department, Towson University, USA.

Tel: 410-704-3067, (410) 337-2923

Citation: Webermann AR, Brand BL, Schielke HJ, Kumar SA, Myrick AC (2017) Depression and Culture in the TOP DD Network Study: Assessing Depressive Symptoms, Self-harm, and Hospitalization among Dissociative Patients across Four Cultures. Acta Psychopathol Vol. 3 No. S1: 39.

\section{Introduction}

\section{Depression and culture in the TOP DD network study: Assessing depressive symptoms, self- harm, and hospitalization among dissociative patients across four cultures}

Depressive symptoms, Non-suicidal Self-injury (NSSI), and suicide attempts are common among individuals with severe dissociative symptoms and Dissociative Disorders (DDs). These difficulties are particularly severe among individuals with complex DDs including dissociative identity disorder (DID) and DD not otherwise specified/other-specified DD (DDNOS/OSDD). The prevalence of DID is estimated at $1 \%$ of community samples and up to $12 \%$ of psychiatric inpatients across multiple countries (Norway, Finland, Switzerland, Turkey, Germany, Netherlands, Canada, United States, and China) [1]. A recent study by Chiu et al. [2] identified $19.5 \%$ of an inpatient sample in Taiwan as meeting criteria for a DD, a prevalence rate comparable to studies conducted with Western inpatient samples. Their findings led them to conclude that DDs present similarly across Eastern and Western societies, including having complex symptom patterns and extensive histories of interpersonal trauma.

Patients with DID have extensive psychiatric comorbidity; for example, 83-96\% have comorbid depressive disorders [3-9]. Individuals with DID typically experienced chronic and severe childhood abuse $[5,10]$. They present with polysymptomatic concerns that are trauma (i.e., dissociation, posttraumatic stress disorder) and non-trauma-related (e.g., depression, anxiety, misuse of substances [5,11]). A majority of those with DID engage in Non-suicidal Self-injury (NSSI) and as many as $86 \%$ attempt suicide $[1,3,5,6,9,12-15]$. In a study of 231 inner city patients consecutively admitted to an outpatient clinic, 
those with a DD were 15 times more likely have made multiple suicide attempts, compared to those with borderline personality disorder, posttraumatic stress disorder, or child abuse histories [4]. The authors concluded, "Dissociative disorders are commonly overlooked in studies of suicidality, but in this population they were the strongest predictor of multiple suicide attempter status." A study of outpatient individuals with DDs found depressive symptoms predicted which patients engaged in NSSI, while dissociative symptoms predicted which patients engaged in either NSSI and/or made suicide attempts [16]. Almost all of these studies have been conducted in the United States. This research indicates the importance of learning more about depression, suicidality, and NSSI among DDs across different cultures.

\section{The Present Study}

The present study assessed depressive symptoms and related behavior among DD community outpatients from the United States (U.S), Canada, Australia, and Norway. Depressive disorders (diagnosed using the fifth edition of the Diagnostic and Statistical Manual of Mental Disorders [DSM-5] [17]), depressive symptoms, number of suicide attempts in the patient's lifetime and recent history, number of self-harm incidents in the patient's recent history, and lifetime and recent psychiatric hospitalizations were examined among participants using both patient and therapist reports. We hypothesized differences in hospitalization given differences in healthcare systems and utilization, with U.S. participants having fewer inpatient days than other countries. In addition, we hypothesized there would be no other differences in depressive symptoms and related behavior among DD patients across cultures, supporting the notion that complex trauma and dissociation has a similar impact regardless of culture.

\section{Methods}

\section{Participants}

DD patients and clinicians were recruited through the Treatment of Patients with Dissociative Disorders (TOP DD) Network study, an online longitudinal educational intervention study of patients with DDs who were diagnosed with either DID or DD not otherwise specified/other-specified DD (DDNOS/OSDD). Both the patient and clinician providing individual psychotherapy to the patient were required to enroll together to ensure that all patients had professional assistance in case of crisis. Data were derived from the screening survey, which determined eligibility to enroll in the study, and the baseline survey that was completed within days of being enrolled in the study. The sample size for the screening survey was $N=265$ and for the baseline survey was $N=237$ (some potential participants did not meet eligibility criteria; others decided not to enroll after completing the screen). The TOP DD Network study included participants from 16 countries, with the largest number of participants from the U.S. (42.3\%, $N=112)$, Norway (27.5\%, $N=73)$, Canada $(6.4 \%, N=17)$, and Australia $(6.4 \%$, $N=17)$. Patient participants were primarily cisgender female (88.6\%), Caucasian (82.1\%), middle-aged (Median=41), and highly educated (50.9\% had at least a college diploma). Patients were primarily diagnosed with DID (63.4\%) (Table 1).

\section{Measures}

\section{Patient NSSI, suicide attempts, and hospitalizations}

Therapists were asked about their patient's history of suicide attempts, self-harm, and hospitalizations. Items queried their patients' number of lifetime, last year, and last six-month suicide attempts; number of self-harm incidents over the last six-months; number of lifetime and last six month psychiatric hospitalizations; and number of inpatient days in the last 6 months.

\section{Depressive symptoms}

Patients completed two measures at the screening survey which gauged the frequency of depressive symptoms, including the 10-item major depressive episode subscale of the Perceived Causal Relations Scale (PCR; $[18,19])$ and the DSM-5 CrossCutting Symptom Measure for Adults (CCSM; [17]). The PCR is a validated measure of patients' attributions regarding the causes and interrelations between their psychiatric symptoms [18]. For the present study, we examined patient responses to the 10 -item Major Depressive Episode (MDE) symptom scale. The CCSM is an emerging measure included in the DSM-5. We examined patients responses to the two depression domain items, which assess loss of pleasure and interest in daily activities and depressed mood.

\section{Depressive disorders}

Therapists were asked on the screening survey if their patients were diagnosed with comorbid depressive disorders, including Disruptive Mood Dysregulation Disorder (DMDD), major depressive disorder, persistent depressive disorder (dysthymia), premenstrual dysphoric disorder, and "other depressive disorder," which are the depressive disorders included in the DSM-5 [17].

\section{Analyses}

The Kruskal-Wallis $H$ Test, a Multivariate Analysis of Variance (MANOVA), and chi-square tests of association were used to assess differences in depressive symptoms and related behavior in participants across four cultures. Kruskal-Wallis was used for patient NSSI, suicide attempts, and hospitalizations because the variables were significantly skewed and kurtotic given the wide range of patient NSSI, suicidality, and hospitalization days. MANOVA was used to assess differences in three depressive symptom measures (the PCR major depressive episode subscale, and two CCSM items) across an independent variable (country) with four levels (Norway, U.S, Australia, and Canada). Chi-square tests of association were used to gauge association between two categorical variables: absence or presence of depressive disorder, and country (U.S, Australia, and Canada; Norway was excluded since it does not use the DSM-5 for diagnoses). The Bonferroni corrected critical $\alpha$ for multiple hypothesis testing $=0.003$.

\section{Results}

Correlations were run to assess associations between patient age, gender, and the study outcomes, and all were non-significant at $p<0.003$. 
Table 1 Participants' countries.

\begin{tabular}{|c|c|}
\hline Country & Frequency \% $(n)$ \\
\hline United States & $42.3(112)$ \\
\hline Norway & $27.5(73)$ \\
\hline Australia & $6.4(17)$ \\
\hline Canada & $6.4(17)$ \\
\hline Sweden & $4.9(13)$ \\
\hline United Kingdom & $4.5(12)$ \\
\hline India & $3.1(8)$ \\
\hline Netherlands & $2.3(6)$ \\
\hline Other $^{1}$ & $2.6(8)$ \\
\hline
\end{tabular}

Note: ${ }^{1}$ Other countries in the sample included Romania $(n=2)$, and one participant each from Belgium, Ireland, Israel, Lithuania, New Zealand, and Spain.

\section{Patient depression-related behavior (NSSI, suicide attempts, hospitalizations)}

Significant differences were found in number of patient lifetime psychiatric hospitalizations across cultures as reported by clinicians, $\chi^{2}(3)=22.506, p<0.001$. Australian clinicians reported a significantly greater number of lifetime hospitalizations among their patients compared to American clinicians ( $M$ ranks 64.80 vs. 33.38, $p<0.001$ ) and Canadian clinicians ( $M$ ranks 14.50 vs. $6.50, p=0.002)$. Norwegian clinicians reported a significantly greater number of lifetime hospitalizations among their patients compared to American clinicians ( $M$ ranks 66.05 vs. 47.52, $p=0.002$ ). No differences in lifetime hospitalizations were found in reports of American and Canadian clinicians ( $p=0.65)$, Canadian and Norwegian clinicians $(p=0.04)$, and Australian and Norwegian clinicians $(p=0.121)$.

Significant differences were also found in number of hospitalizations in the last six months across cultures as reported by clinicians, $\chi^{2}(3)=41.211, p<0.001$. Australian clinicians reported a significantly greater number of hospitalizations among their patients within the prior six months compared to American clinicians ( $M$ ranks 73.18 vs. $49.28, p<0.001$ ). Norwegian clinicians reported a significantly greater number of hospitalizations within the prior six months compared to American clinicians ( $M$ ranks 92.46 vs. $58.30, p<0.001$ ) and Canadian clinicians ( $M$ ranks 37.70 vs. $20.38, p<0.001)$. No differences in six month hospitalizations were found between the American and Canadian clinicians $(p=0.667)$, Canadian and Australian clinicians $(p=0.022)$, and Australian and Norwegian clinicians ( $p=0.891)$.

There were no significant differences across cultures in the number of days of hospitalization in the prior six months $(p<0.01)$, lifetime suicide attempts $(p<0.05)$, last year suicide attempts $(p<0.05)$, suicide attempts in the prior six months $(p=0.14)$, or self-harm in the prior six months $(p=0.55)$.

\section{Depressive symptoms and disorders}

There was not a significant difference in depressive symptoms (as assessed by the PCR and CCSR) across cultures ( $p=0.34$ ). Additionally, there was not a significant relationship between the three cultures (Australia, U.S, and Canada) and DMDD ( $p=0.56)$, major depressive disorder $(p=0.72)$, persistent depressive disorder (dysthymia; $p=0.30$ ), premenstrual dysphoric disorder $(p=0.56)$, and other depressive disorder $(p=0.34)$.

\section{Discussion}

The present study assessed depressive symptoms and behaviors in DD patients receiving outpatient treatment across the U.S, Australia, Canada, and Norway. Ashypothesized, theonlysignificant differences were in psychiatric hospitalizations, with American and Canadian patients consistently demonstrating significantly fewer psychiatric lifetime and last six-month hospitalizations compared to Norwegian and Australian DD patients. Differences in suicide attempts were not found after applying Bonferroni alpha corrections. There were no cross-cultural differences in NSSI, depressive symptoms, or types of depressive disorders. Our findings regarding rates of hospitalization are likely due to cross-cultural differences in health care utilization and systems, rather than to phenomenology related to depression. The lack of cross-cultural differences in individuals with DDs may also suggest that trauma and dissociation have a consistent link with depressive symptomatology, independent of culture. Consistent with the findings of the present study, dissociation has been linked to NSSI, depression and suicidality in among DDs and in non-DD clinical populations across many cultures and countries. Greater suicidality has also been found among DD patients in the U.S, Turkey and the Netherlands, among others $[4,7,20]$. In a general population study conducted in Finland, Maaranen et al. [21] found that dissociation was nine-fold more likely among depressive individuals and more than four-fold higher among suicidal individuals than among individuals who were not high in dissociation. Suicidality was independently associated with dissociation, even after adjusting for covariates including depression. In a three-year follow up [22], the individuals who were high in dissociation at both time points showed an increase in depression and suicidal ideation over time. Those who were not initially high in dissociation but became high at follow-up evidenced an increase in depressive symptoms whereas recovery from high dissociation was associated with improvement in depression. In a 10-year prospective study of U.S. patients with borderline personality disorder, the severity of dissociation and a diagnosis of major depression were among six out of 11 predictors that predicted self-harm [23]. Future research should investigate other factors which relate to suicidality in clinical populations to gauge their relevance with dissociative patients, such as sleep disturbances and a family history of suicidality [24-26].

An implication of the present study is that dissociation generally, as well as DDs in particular, are strongly linked with depression, NSSI, and suicidality; however, dissociation and DDs remain underrecognized and under-treated $[4,7,10,27]$. Clinicians need to be trained in assessing and treating dissociation, routinely assess for dissociation in all clinical settings, and be aware of the risk of suicidality, NSSI, and comorbid depression among individuals high in dissociation. Recent studies have demonstrated evidence that treatment of DDs can result in significant reductions in depressive symptoms and suicidality. A meta-analysis of DD treatment studies found significant reductions in depressive symptoms and suicidality among individuals with DDs [28]. 
Additionally, the first TOP DD study, a naturalistic international longitudinal study of DD patients and therapists, found that over 30 months of individual therapy, patients demonstrated significant reductions in self-harm, hospitalizations, and trauma symptoms, as well as improved global functioning [29].

\section{Conclusion}

The study's most notable limitation was that sample sizes in

\section{References}

1 Foote B (2013) Dissociative identity disorder: Epidemiology, pathogenesis, clinical manifestations, course, assessment, and diagnosis. In: Spiegel D, Hermann R (eds.) UpToDate, Waltham, MA, USA.

2 Chiu CD, Tseng MCM, Chien YL, LiaoSC, Liu CM, et al. (2017) Dissociative disorders in acute psychiatric inpatients in Taiwan. Psychiatry Res 250: 285-290.

3 Foote B, Park J (2008) Dissociative identity disorder and schizophrenia: differential diagnosis and theoretical issues. Curr Psychiatry Rep 10: 217-222.

4 Foote B, Smolin Y, Neft DI, Lipschitz D (2008) Dissociative disorders and suicidality in psychiatric outpatients. J Nerv Ment Dis 196: 29-36.

5 Ross CA, Miller SD, Reagor P, Bjornson L, Fraser GA, et al. (1990) Structured interview data on 102 cases of multiple personality disorder from four centers. Am J Psychiatry 147: 596-601.

6 Ross CA, Norton GR (1989) Differences between men and women with multiple personality disorder. Hosp Commun Psychiatry 40: 186-188.

7 Şar V, Akyuz G, Dogan O (2007) Prevalence of dissociative disorders among women in the general population. Psychiatry Res 149: 169176.

8 Saxe GN, Van der Kolk BA, Berkowitz R, Chinman G (1993) Dissociative disorders in psychiatric inpatients. Am J Psychiatry 150: 1037-1042.

9 Yargiç L, Şar V, Tutkun H, Alyanak B (1998) Comparison of dissociative identity disorder with other diagnostic groups using a structured interview in Turkey. Compr Psychiatry 39: 345-351.

10 Brand BL, Classen CC, Lanius R, Loewenstein RJ, McNary SW, et al. (2009) A naturalistic study of dissociative identity disorder and dissociative disorder not otherwise specified patients treated by community clinicians. Psychol Trauma 1: 153-171.

11 http://www.isst-d.org/downloads/GUIDELINES_REVISED2011.pdf

12 Coons PM, Milstein V (1990) Self-mutilation associated with dissociative disorders. Dissociation: Progress in the Dissociative Disorders 3: 81-87.

13 Loewenstein RJ, Putnam FW (1990) The clinical phenomenology of males with MPD: A report of 21 cases. Dissociation 3: 135-143.

14 Putnam FW, Guroff JJ, Silberman EK, Barban L, Post RM (1986) Clinical phenomenology of multiple personality disorder: review of 100 recent cases. J Clin Psychiatry 47: 285-293.

15 Saxe GN, Chawla N, Van Der Kolk B (2002) Self-destructive behavior in patients with dissociative disorders. Suicide Life Threat Behav 32 313-320. many countries were too small to be included in the analyses. Additionally, there were no participants from Africa and very few from Asia, limiting the geographic generalizability of our conclusions. Overall, the finding of a lack of cross-cultural differences in depression-related symptomatology in individuals with DDs suggests that dissociation, and possibly the trauma that is typically linked to dissociation, may be associated with depressive symptomatology, regardless of culture.

16 Webermann AR, Myrick AC, Taylor CL, Chasson GS, Brand BL (2016) Dissociative, depressive, and PTSD severity as correlates of nonsuicidal self-injury and suicidality in dissociative disorder patients. J Trauma Dissociation 17: 67-80.

17 American Psychiatric Association (2013) Diagnostic and statistical manual of mental disorders ( $5^{\text {th }}$ edn.). Arlington VA: American Psychiatric Publishing.

18 Frewen PA, Allen SL, Lanius RA, Newfeld RW (2012) Perceived causal relations: novel methodology for assessing client attributions about causal associations between variables including symptoms and functional impairment. Assessment 19: 480-493.

19 Frewen PA, Schmittmann VD, Bringmann LF, Borsboom D (2013) Perceived causal relations between anxiety, posttraumatic stress and depression: extension to moderation, mediation, and network analysis. Eur J Psychotraumatol 30: 4.

20 Boon S, Draijer N (1993) Multiple personality disorder in the Netherlands: a clinical investigation of 71 patients. Am J Psychiatry 150: 489-494.

21 Maaranen P, Tanskanen A, Honkalampi K, Haatainen K, Hintikka J, et al. (2005) Factors associated with pathological dissociation in the general population. Aust N Z J Psychiatry 39: 387-394.

22 Maaranen P, Tanskanen A, Hintikka J, Honkalampi K, Haatainen K et al. (2008) The course of dissociation in the general population: $A$ 3-year follow-up study. Compr Psychiatry 49: 269-274.

23 Zanarini MC, Frankenbur FR, Weingeroff JL, Reich DB, Fitzmaurice GM, et al. (2011) The course of substance use disorders in patients with borderline personality disorder and axis II comparison subjects: a 10-year follow-up study. Addiction 106: 342-348.

24 Sosso FAE (2017) Negative involvement of the working environment in the occurrence of cognitive disorders. Transl Biomed 8: 2.

25 Sosso FAE (2016) Neurocognitive game between risk factors, sleep and suicidal behaviour. Sleep Sci 10: 41-46.

26 Sosso FAE, Raouafi S (2016) Brain disorders: correlation between cognitive impairment and complex combination. J Ment Health Fam Med 12: 215-222.

27 Şar V, Yargiç LI, Tutkun H (1996) Structured interview data on 35 cases of dissociative identity disorder in Turkey. Am J Psychiatry 153: 1329.

28 Brand BL, Classen CC, McNary SW, Zaveri P (2009) A review of dissociative disorders treatment studies. J Nerv Ment Dis 197: 646654.

29 Brand BL, McNary SW, Myrick AC, Classen CC, Lanius R, et al. (2013) A longitudinal naturalistic study of patients with dissociative disorders treated by community clinicians. Psychol Trauma 5: 301-308. 